\title{
How does the Facebook Algorithm rank the contents according to the interaction buttons?
}

Pócs D, Department of Behavioral Science, Faculty of Medicine, University of Szeged.

\section{Objectives}

This research aimed at understanding how social media contents are ranked by the Facebook Algorithm according to interaction buttons. The correlations between Facebook users' interactions and the organic reach of the given content can help to gain this aim

\section{Methods}

We included 1025 non-paid, Facebook posts ( $N=1025)$.

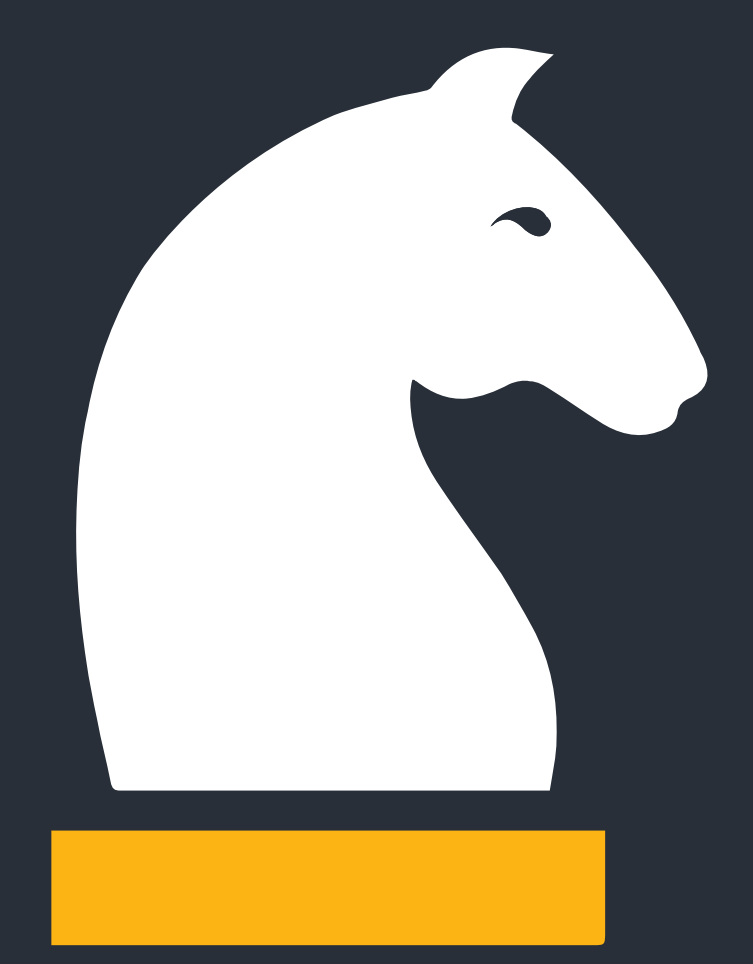
We collected the following data in the post level. "Organic reach" is the number of people who saw the given non-paid content. The organic reach consisted of "fan reach" and "non-fan reach" according to previous "page like" activity. The investigated interaction data was the following: "Facebook reactions" (e.g., "like"); "shares"; "comments"; "clicks"; and "negative Facebook interactions".

\section{Results}

A significant negative correlation was found between organic reach and "like" reaction. We observed the strongest significant positive correlations of organic reach with "comments", "haha" and "love" reactions. Furthermore, fan reach correlated positively with "comments", "haha" and "love" reactions, while non-fan reach correlated positively with "shares" and "clicks".

\section{Conclusions}

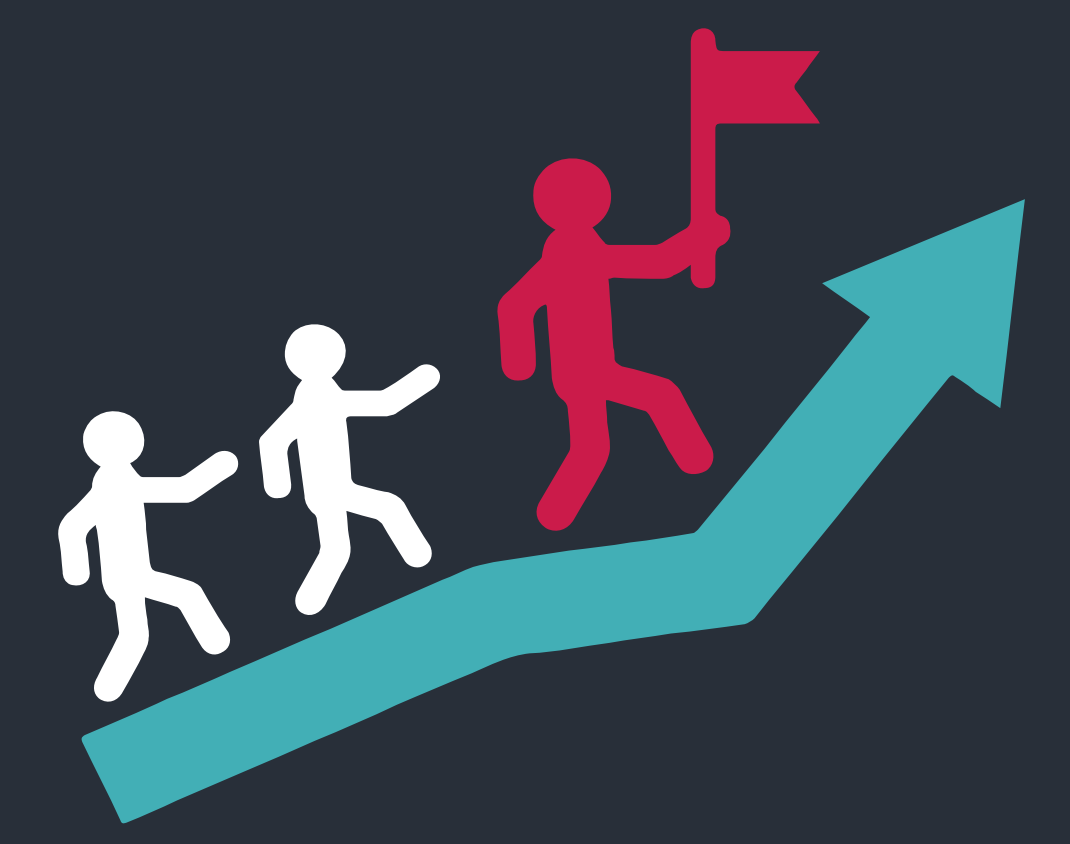

This exploratory research offers an important insight into the Facebook content ranking for public health professionals who design Facebook-based interventions.

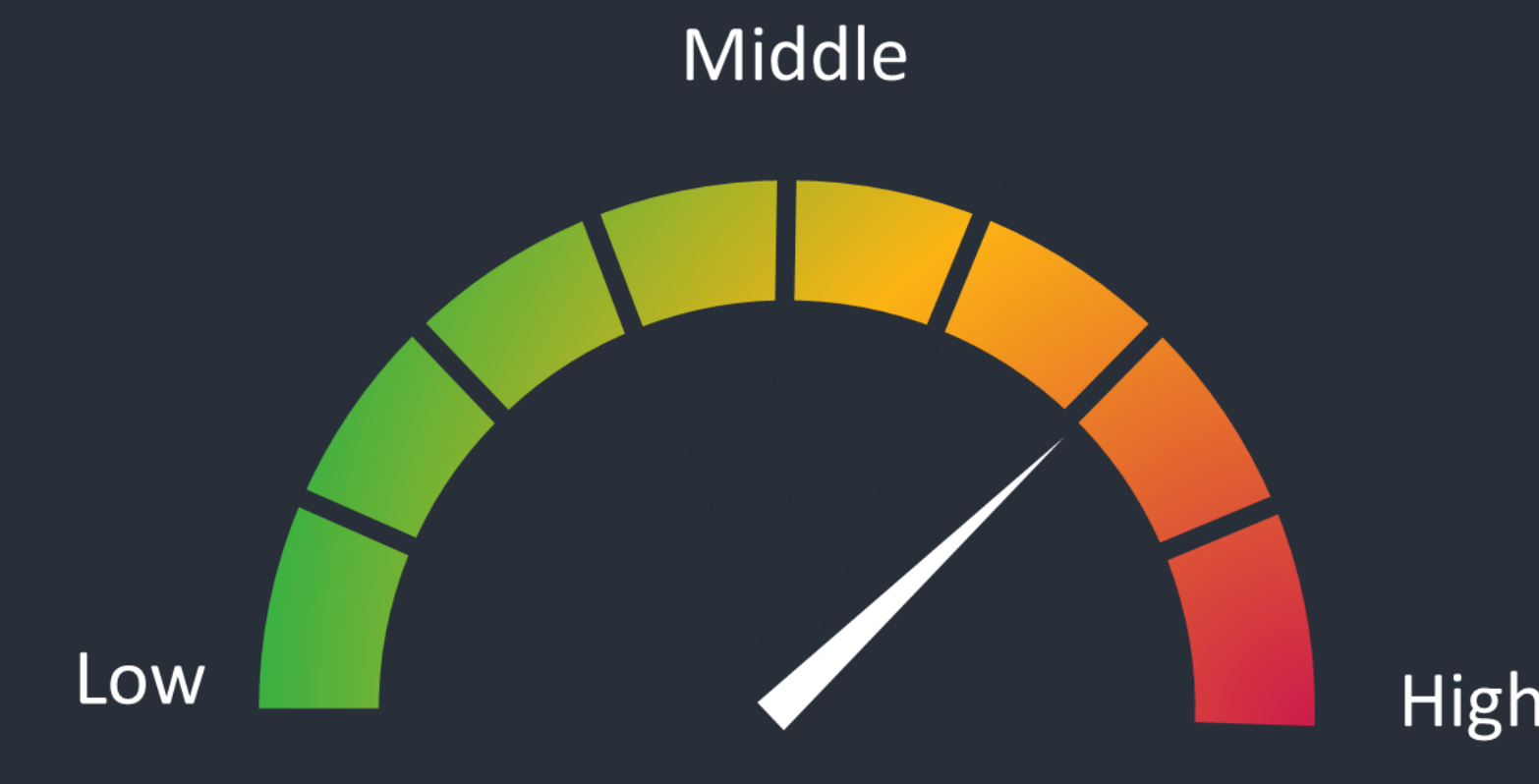

Some interactions on a post level can have higher priority in content ranking: "comments", "haha", "love", and "sad" reactions.

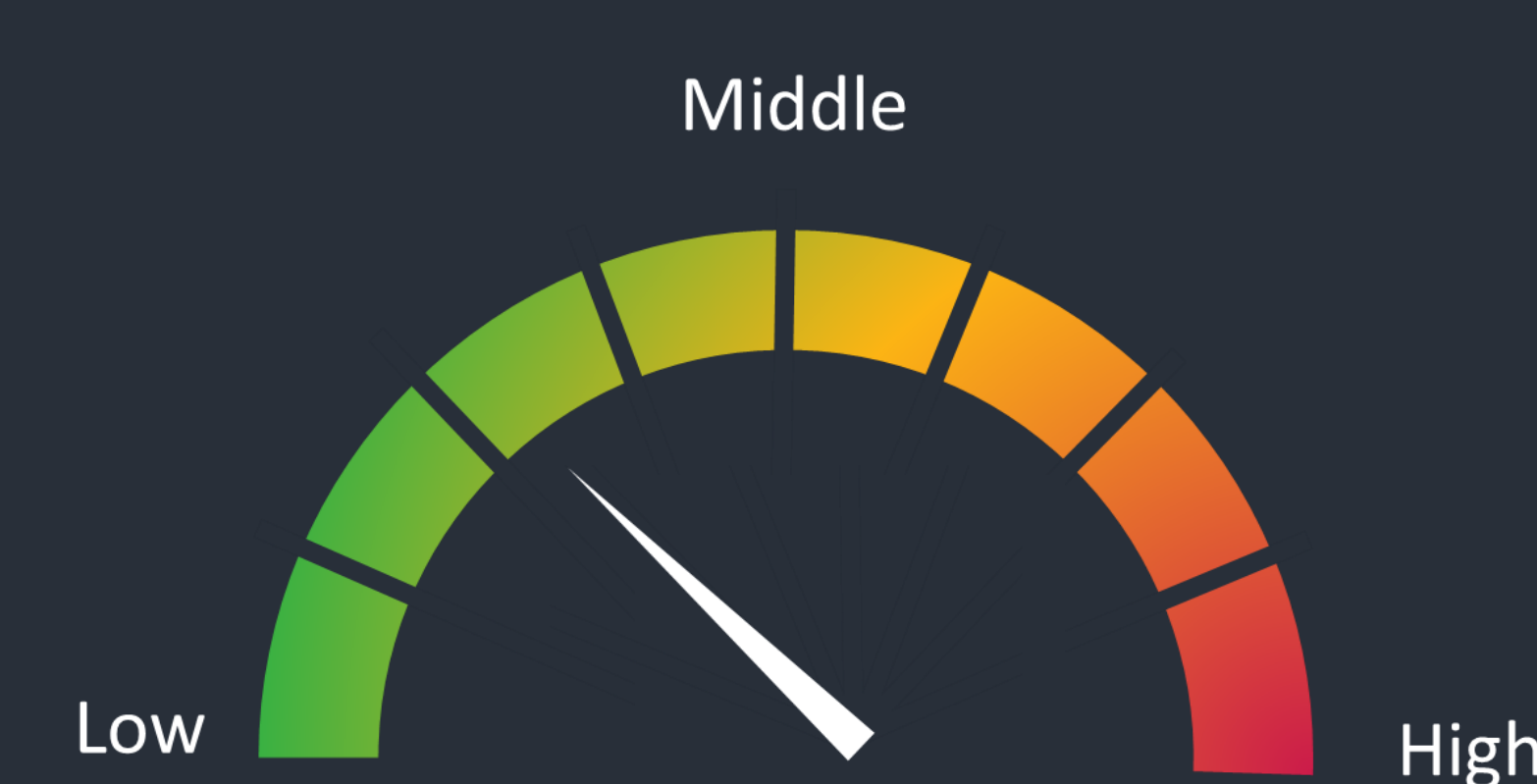

This study has shown that "like" reactions and "shares" can have a lower priority in content ranking.

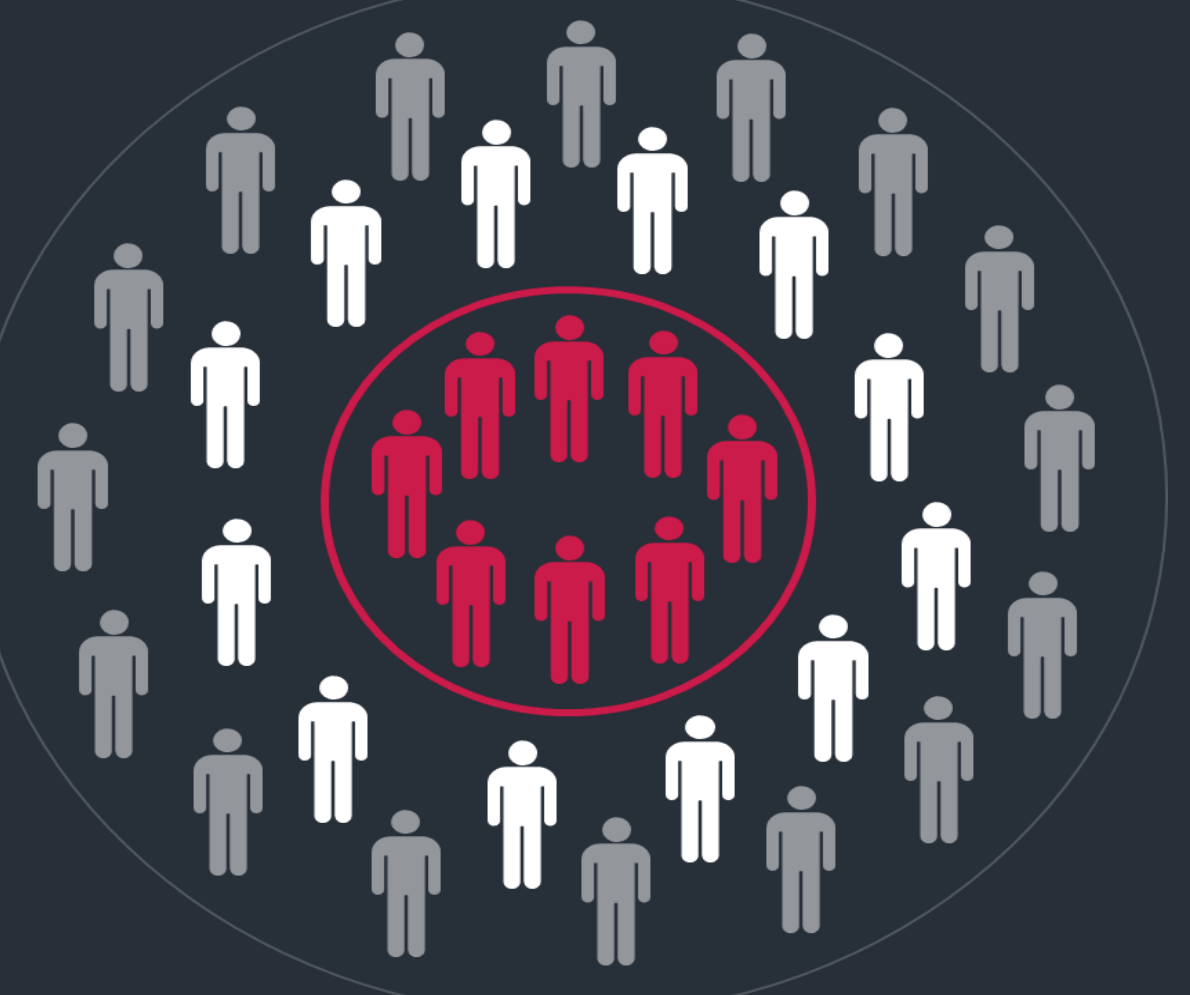

Our results suggest that a further categorization to

("haha" and "love" reactions, "comments") and non-fan-specific interactions ("shares" and "clicks") is needed.

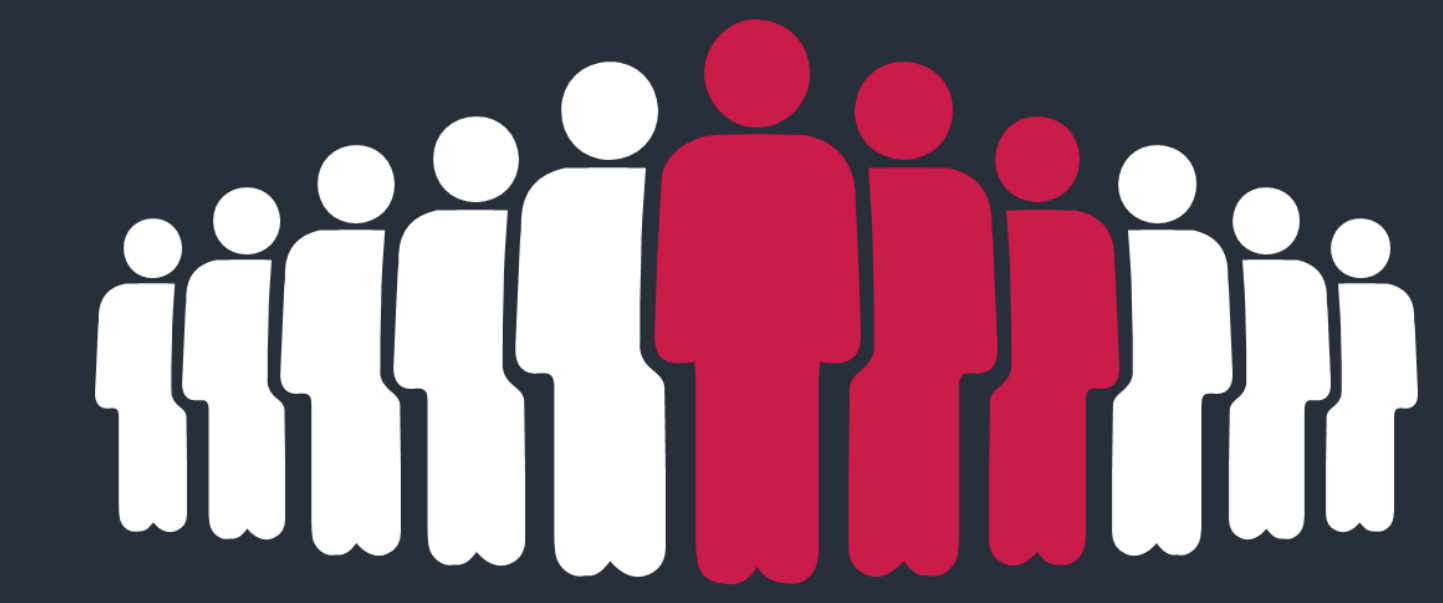

„Shares" can generate more non-fans, however, "shares" can result in fewer fans. 